\title{
Somatic distress, mental health and psychological resilience among cancer patients during the pandemic
}

\author{
Eser Sağaltıc1 ${ }^{1}$ Özcan Sönmez ${ }^{2}$, Ebru Karci³ ${ }^{3}$ Șengül Kocamer Şahin ${ }^{4 *}$, Arzu Erturk ${ }^{5}$ \\ ${ }^{1}$ Department of Psychiatry, Bagcilar Research and Training Hospital, University of Health Sciences, Turkey \\ ${ }^{2}$ Department of Internal Medicine, Bagcilar Research and Training Hospital, University of Health Sciences, \\ Turkey \\ ${ }^{3}$ Department of Medical Oncology, Bagcilar Research and Training Hospital, University of Health Sciences, \\ Turkey \\ ${ }^{4}$ Department of Psychiatry, Faculty of Medicine, Gaziantep University, Turkey \\ ${ }^{5}$ Department of Social Work, School of Health, Düzce University, Turkey \\ *Corresponding author, e-mail: snglkcmr@hotmail.com
}

\begin{abstract}
We aimed to investigate the somatic distress and psychological symptoms levels of cancer patients, and analyze the influencing factors on somatic distress during COVID-19 pandemic. This crosssectional study included 216 eligible cancer patients. The Patient Health Questionnaire-15, Posttraumatic Stress Disorder (PTSD) Checklist for DSM-5, The Depression, Anxiety and Stress Scale21, the Brief Resilience Scale, the Stressful Life Events List due to Pandemic were administered to the participants. The moderate to severe somatic distress rate was \% 38 and probable PTSD rate was $20.4 \%$. Depression, anxiety and stress symptoms were $36.1 \%, 49.1 \%$ and $45.4 \%$, respectively, from mild to extremely severe. There were substantial association between somatic symptoms severity and high PTSD, anxiety, depression, and stress symptoms levels. Low education, high anxiety levels, high experience stressful life events, and low psychological resilience predicted high somatic distress. This study demonstrates the high risk of somatic distress, PTSD, depression, anxiety and stress in patients with cancer during the pandemic. In addition, somatic distress may indicate high levels of psychological symptoms, high experience stressful life events, and low psychological resilience. It underscores the need to assess psychological status during the pandemic, especially those with high level somatic symptoms.
\end{abstract}

Keywords: COVID-19, Cancer, Somatic Distress, Post-traumatic Stress, Depression.

How to Cite: Sağaltıcı, E., Sönmez, Ö., Karcı, E. Şahin, Ş. K., Erturk, A. (2021). Somatic distress, mental health and psychological resilience among cancer patients during the pandemic. International Research in Counseling and Education, 5 (2): pp. 116-127, DOI: https://doi.org/10.24036/00435za0002

\section{Introduction}

Corona Virus Disease 2019 (COVID-19) has been detected for the first time in December 2019 in Wuhan, China as a novel pneumonia causing respiratory tract infection (Wang et al., 2020). COVID-19, indicating person-to-person transmission and an asymptomatic course has rapidly spreaded out to the whole world (Chan et al., 2020). Most people infected with the Covid-19 virus have been experienced mild to moderate respiratory syndrome and have been recovered without special treatment. Developing serious consequences is a greater risk for the elderly, those with underlying medical conditions such as cardiovascular diseases, diabetes, chronic respiratory issues, and cancer (Wu \& Mc Googan, 2020; Yang et al., 2020). The COVID-19 pandemic has created an unprecedented change in the lives of people worldwide, especially in patients with chronic diseases. Those with cancer may be particularly vulnerable to more severe disease due to their immunocompromised status from the underlying malignancy itself, as well as decreased immunity from cancer-directed treatments, additional medical comorbidities, and poor nutritional status (Gonzalez et al., 2020).

A few recent studies that evaluated psychological symptoms in oncology patients during the COVID-19 pandemic (Juanjuan et al., 2020; Wang et al., 2020; Romito et al., 2020; Miaskowski et al., 2020). Occurrence rates for depression and anxiety ranged from $9.3 \%$ to $31.0 \%$ and from $8.9 \%$ to $36.0 \%$, respectively (Juanjuan et 
al., 2020; Wang et al., 2020; Romito et al., 2020). In a study conducted on 187 oncology patients have been reported, rate of with probable post-traumatic stress disorder (PTSD) 31.6\% (Miaskowski et al., 2020). But, Somatic distress has not been investigated yet in cancer patients during the pandemic. Somatic distress presentation in cancer is a complex area because of the overlapping of physical disease and treatment-related mechanisms and possible psychosocial mechanisms, and it is that needs investigation (Grassi L, Caruso R \& Nanni MG, 2013) Previous studies have shown that heightened distress levels can lead to adverse outcomes in cancer patients including decreased satisfaction with care and noncompliance with treatment, low survival rates, desire to accelerate death, and poor quality of life for both patients and their relatives (Breitbart et al., 2000; Anuk et al., 2019).

Looking at the new, unrecognized and early unpredictable and uncontrollable pandemic process, it may be much more intense than the stress factors in daily life, it creates a common threat perception, there is a lack of information about the process, it includes social isolation and quarantine processes, in addition to the fact that there is uncertainty about the future, social and economic effects are evident, it is observed that it is distinguished by the fact that it creates a massive stress all over the world (Eser, 2020). It can be thought that the special population such as cancer patients will be affected more by this mass stress and its effect on them may cause more severe consequences. Therefore, it will be of great importance to define the psychological symptoms that may occur in cancer patients during the pandemic. For these reasons, this study aimed to a) estimate the levels of somatic, PTSD, depression, anxiety, and stress symptoms and b) investigate the relationship between somatic distress and demographic characteristics, psychological symptoms, stressful life events, and psychological resilience among the cancer patients during the pandemic. In this way, we aimed to shed light on the measures to be taken to prevent somatic distress and worse mental problems of cancer patients during the pandemic.

\section{Method}

\section{Sample and Procedure}

This cross-sectional study was conducted in XXXXXXXXX, between 15 June and 15 October 2020 . This study included consecutively selected 216 eligible cancer patients, who receiving treatment or visiting the oncology center for routine follow-up examinations. To be eligible for study participation, patients had to be (a) diagnosed with any cancer type, (b) aged between 18 and 75 years, (c) able to complete questionnaire in Turkish language, and (d) able to provide informed consent. Reasons for exclusion were pregnancy, illiteracy, substance abuse, declared inability to complete questionnaires, serious psychiatric disorders that hindered judgement, and patients with any issues interfering with giving informed consent. The aim of the study was explained to the participants and invited to voluntarily participate, and informed consent forms were obtained before they were included in the study. This research was approved by the XXX XXXXXX ethical committee (reference 2020.06.1.11.082) and was conducted in accordance with the Declaration of Helsinki. The study protocol was also approved by the Turkish Ministry of Health, General Directorate of Health Services (reference ES-2020-0507T13_40_22). The Patient Health Questionnaire-15 (PHQ-15), Posttraumatic Stress Disorder Checklist for DSM-5 (PCL-5), The Depression, Anxiety and Stress Scale-21(DASS-21), the Brief Resilience Scale (BRS), the Stressful Life Events List due to Pandemic and, demographic and clinical characteristics were administered to the participants with face to face interweaving by author $\mathrm{AE}$ who senior social worker trained for the study.

\section{Measures}

\section{Demographic and clinical characteristics form.}

Age, gender, marital status, education, employment status, economic well-being, comorbidity (hypertension, diabetes, coronary heart disease, chronic obstructive lung disease, chronic kidney disease, and other), and history of psychiatric disorders were assessed via self-report. Clinical characteristics included type of cancer, time since diagnosis, types of treatment currently or previously received, present treatment, and metastasis; this information was obtained from participants' medical charts.

\section{Stressful Events List due to Pandemic.}

The scale was prepared by authors, using the Stressful Life Events Screening Questionnaire (Wolfe et al., 1996) and review of the literature, and used to measure the stressful life event burden during the pandemic. The scale, answers are 0 -no, 1 -yes consisting of 18 questions and total scores ranging from 0 to 18. Higher scores on the scale are associated with stressful event burden. Cronbach's alpha internal consistency coefficient was determined as 0.73 . A positive and significant relationship was found between the total score of the scale and depression $(\mathrm{r}=.35)$, anxiety $(\mathrm{r}=.32)$, stress $(\mathrm{r}=.32)$, PCL-5 total score $(\mathrm{r}=.41)$, and PHQ-15 total score $(\mathrm{r}=$ .38) (see Supplementary Table 1 ). 


\section{Patient Health Questionnaire (PHQ-15)}

Somatic symptoms were measured using the PHQ-15 scale, which includes the 15 most common somatic symptoms. This scale assesses how much participants were bothered by health symptoms such as "abdominal pain", "back pain" on a scale of $0=$ not bothered at all to $2=$ bothered a lot. A total somatic symptom score was calculated, with possible scores ranging from 0 to 30 , and scores scaled to account for the additional item asked only of female participants relating to menstrual pain and problems. Higher scores on the PHQ-15 are strongly associated with somatic symptoms. Somatic symptom severity were calculated in four categories based on the PHQ-15 score 'minimal' (0-4), 'mild' (5-9), 'moderate' (10-14), or 'severe' $(\geq 15)$ ( Kroenke, Spitzer \& Williams, 2002). The Turkish validity-reliability study was conducted by Yazıcı Güleç et al. (2012) (Yazıcı et al., 2012). In this study, Cronbach's alpha of the PHQ-15 was 0.86 .

\section{Post-traumatic stress disorder checklist for DSM-5 (PCL-5)}

The PCL-5 is a 20-item measure that assesses PTSD symptomatology: intrusions, avoidance, negative alterations in cognitions and mood (NACM), and hyperarousal. Participants responded to the items on 5-point Likert-type scales ( $0=$ not at all to $4=$ extremely) in relation to their experience of COVID-19 outbreak, with total scores ranging from 0 to 80 (Weathers et al., 2013). The Turkish version of PCL-5 was used, which has been shown to be reliable and valid. In this study, used $\geq 47$ as a cut-off point to diagnose possible PTSD (Boysan et al., 2017). Among the current sample, the PCL-5 and subscales evidenced a Cronbach's alpha of $\alpha=.96$ for PCL$5, \alpha=.89$ for intrusions, $\alpha=.87$ for avoidance, $\alpha=.91$ for NACM, and $\alpha=.92$ for hyperarousal.

\section{The Depression Anxiety Stress Scales-21 (DASS-21)}

DASS-21 is a 21-item, self-report questionnaire designed to measure the severity of a range of depression, anxiety and stress symptoms. Each item of the DASS corresponds to one of the three subscales (depression, anxiety, and stress) with 7 items per subscale. The scale is a 4-point Likert from 0 (never) to 3 (almost always) and evaluates symptoms from last week (Lovibond \& Lovibond SH, 1995; Akin A \& Cetin B, 2007). DASS-21 raw scores were doubled for comparability to full-length (42 items) DASS scores. The depression score results were classified as normal (0-9), mild (10-12), moderate (13-20), severe (21-27), and extremely severe (28-42). The anxiety score results are classified as normal (0-6), mild (7-9), moderate (10-14), severe (15-19), and extreme severe (20-42). The stress score results were classified as normal (0-10), mild (11-18), moderate (19-26), severe (27-34), and extreme severe (35-42)[21]. Among the current sample, the DASS-21and subscales evidenced a Cronbach's alpha of $\alpha=.93$ for DASS-21, $\alpha=.86$ for depression, $\alpha=.78$ for anxiety and $\alpha=.83$ for stress.

\section{Brief Resilience Scale (BRS)}

The BRS includes six items. The respondents were asked to indicate how well each statement described their behaviour and actions on a 5-point Likert-type scale, ranging from " 1 " = does not describe me at all to "5" = describes me very well. As Item 2 (I have a hard time making it through stressful events), Item 4 (It is hard for me to snap back when something bad happens) and Item 6 (I tend to take a long time to get over set-backs in my life) were reverse-coded, the data collected were recoded prior to analysis. High scores obtained from the scale after the items coded in reverse order are translated in the scale indicate high psychological resilience (Smith et al., 2008). The factor loads for the items were found between 0.68 and 0.91 in Turkish sample (Dogan, 2015). In this study, Cronbach's alpha of the BRS was 0.69.

\section{Statistical Analysis}

Descriptive statistics were presented in median values and interquartile ranges (IQR) (25\% to $75 \%$ ) for the quantitative variables, and frequencies and percentages for the categorical variables. Normality tests were carried out by using one-sample Kolmogorov-Smirnov and Shapiro-Wilk tests and through histogram graphs.The Mann-Whitney U test was utilized for comparing the continuous variables among two groups. Kruskal-Wallis test was used to evaluate possible differences between the more than two groups and the level of significance was determined after the Dunn multiple comparison tests with Bonferroni correction test. Multiple linear regression model was used with backward elimination technique to investigate potentially predictive factors for the somatic distress. The variables evaluated were determined as significant variables derived from our results and literature review, in accordance with clinical experience. The variables used for the models are as follows; age (years), gender, education levels (years), additional chronic disease, history of psychiatric disorders, present treatment (any treatment, follow-up), metastasis anxiety, stressful life events, BRS, and intrusions. The tests for assumptions-linearity, homoscedasticity, and multicollinearity were carried out by the authors (assumptions met). All the analyses were 2-sided with alpha of 0.05 , and performed with SPSS statistical software (IBM SPSS Statistics for Windows, Version 26.0. Armonk, NY: IBM Corp.). 


\section{Results and Discussion}

\section{Sociodemographic and Clinic Characteristics}

The demographic-clinical characteristics of participants are summarized in Table 1.125 (57.9\%) of 216 participants were female and 168 (77.8\%) were married. The median age was 52 years (IQR= 45 to 61 years), median education levels was 5 years (IQR= 5 to 8 years), and time since current diagnosis was 18 months (IQR= 7 to 36 months).

Table 1. Demographic and clinical characteristics

\begin{tabular}{|c|c|c|}
\hline Variables & n (\%)/Median (IQR) & {$[95 \% \mathrm{CI}]$} \\
\hline \multicolumn{2}{|l|}{ Gender } & {$[49.5-54.0]$} \\
\hline Female & $125(57.9)$ & [51.4-64.3] \\
\hline Male & $91(42.1)$ & [35.7-48.6] \\
\hline \multicolumn{3}{|l|}{ Marital status } \\
\hline Married & $168(77.8)$ & {$[72.7-83.3]$} \\
\hline Single & $24(11.1)$ & {$[6.9-15.3]$} \\
\hline Widowed/Divorced & $24(11.1)$ & {$[7.0-15.3]$} \\
\hline Education, years & $5(5-8)$ & {$[5.0-5.0]$} \\
\hline Literate & $40(18.5)$ & [13.4-24.1] \\
\hline Primary education & $125(57.9)$ & [51.9-64.4] \\
\hline High school and above & $51(23.6)$ & {$[18.1-29.6]$} \\
\hline \multicolumn{3}{|l|}{ Employment Status } \\
\hline Employed & $23(10.6)$ & [6.9-14.8] \\
\hline Not Employed & $193(89.4)$ & [85.2-93.1] \\
\hline \multicolumn{3}{|l|}{ Household economic situation } \\
\hline Bad & $124(57.4)$ & [50.9-64.4] \\
\hline Average & $90(41.7)$ & [34.7-47.7] \\
\hline Good or very good & $2(0.9)$ & {$[0.0-2.3]$} \\
\hline \multicolumn{3}{|l|}{ Additional Chronic Disease } \\
\hline No & $128(59.3)$ & [52.3-65.7] \\
\hline Yes & $88(40.7)$ & [34.3-47.7] \\
\hline \multicolumn{3}{|l|}{ History of Psychiatric Disorders } \\
\hline No & $168(77.8)$ & [72.2-82.9] \\
\hline Yes & $48(22.2)$ & {$[17.1-27.8]$} \\
\hline \multicolumn{3}{|l|}{ Cancer type } \\
\hline Breast & $79(36.6)$ & [30.1-43.1] \\
\hline Esophageal/gastrointestinal & $59(27.3)$ & [21.3-32.9] \\
\hline Lung & $17(7.9)$ & {$[4.2-11.6]$} \\
\hline Gynecologic & $15(6.9)$ & {$[3.7-10.6]$} \\
\hline Head and neck & $9(4.2)$ & [1.9-6.9] \\
\hline Sarcoma & $7(3.2)$ & {$[0.9-6.0]$} \\
\hline Others & $30(13.9)$ & {$[9.7-18.5]$} \\
\hline \multicolumn{3}{|l|}{ Current or completed treatment } \\
\hline Chemotherapy & $62(28.7)$ & {$[27.2-35-2]$} \\
\hline Surgery & $3(1.4)$ & {$[0.0-3.2]$} \\
\hline Radiation & $1(0.5)$ & {$[0.0-1.4]$} \\
\hline Hormone therapy & $1(0.5)$ & {$[0.0-1.4]$} \\
\hline Surgery + Chemotherapy & $76(35.2)$ & [28.7-41.7] \\
\hline Chemotherapy + Radiation & $15(6.9)$ & {$[3.7-10.6]$} \\
\hline Surgery + Chemotherapy + Radiation & $53(24.5)$ & [19.0-30.1] \\
\hline No treatment & $5(2.3)$ & {$[0.5-4.6]$} \\
\hline \multicolumn{3}{|l|}{ Present treatment } \\
\hline Any treatment & $147(68.1)$ & [61.6-74.5] \\
\hline Follow-up & $69(31.9)$ & {$[25.5-38.4]$} \\
\hline \multicolumn{3}{|l|}{ Metastasis } \\
\hline No & $169(78.2)$ & [72.7-83.8] \\
\hline Yes & $47(21.8)$ & [16.2-27.3] \\
\hline
\end{tabular}




\begin{tabular}{lcr}
\multicolumn{1}{c}{ Variables } & $\mathbf{n}(\%) /$ Median (IQR) & {$[95 \% \mathrm{CI}]$} \\
\hline $\begin{array}{l}\text { Time since current diagnosis, months } \\
\text { Fear of catching COVID-19 }\end{array}$ & $18(7-36)$ & {$[12-24]$} \\
$\quad$ No & $130(60.2)$ & {$[53.7-66.2]$} \\
$\quad$ Yes & $86(39.8)$ & {$[33.8-46.3]$} \\
Fear of cancer recurrence during pandemic & $148(68.5)$ & {$[62.5-75.0]$} \\
$\quad$ No & $68(31.5)$ & {$[25.0-37.5]$} \\
$\quad$ Yes & & {$[55.1-69.0]$} \\
Fear of not getting appropriate and timely & & {$[31.0-44.9]$} \\
treatment during pandemic & $134(62.0)$ &
\end{tabular}

${ }^{*} \mathrm{CI}$ : Confidence Interval; IQR: Interquartile range $25 \%-75 \%$

Cancer type of 216 patients were 79 (36.6\%) breast, 59 (27.3\%) esophageal/gastrointestinal, 17 (7.9\%) lung, 15 (6.9\%) gynecologic, 9 (4.2\%) head and neck, 7 (3.2\%) sarcoma, and 30 (13.9\%) others. Most of current or completed treatments were 76 (35.2\%) surgery + chemotherapy, 62 (28.7\%) chemotherapy, and 53 (24.5\%) surgery + chemotherapy + radiation. Among 216 participants $147(68.1 \%)$ had any current treatment, and 47 $(21.8 \%)$ had metastasis.

Analyzes of association between demographic-clinical characteristics and stressful life events, BRS, somatic distress, PTSD, depression, anxiety, and stress symptoms were given in Table 2 and Table 3.

\section{Stressful life events list due to the pandemic}

The median of stressful life events due to the pandemic that the participants experienced was 3 (IQR= 1 to 5) (Table 3). Among 216 patients were 9 (4.2\%) diagnosed with COVID-19, 7 (3.2 \%) hospitalized, and 14 (\%6.5) mandatory quarantined. The stressful life events due to the pandemic that participants reported are summarized in Table 2.

\section{Psychometric Properties for Scales and Subscales}

The median of PHQ-15 total score was 8 (IQR= 4 to 12 ). Somatic distress severity levels were $27.8 \%$ minimal, 34.3\% mild, $19.9 \%$ moderate, and $18.1 \%$ severe. The medians of PCL-5 total score, intrusions, avoidance, NACM and hyperarousal were 25 (IQR= 8 to 43$), 6$ (IQR= 2 to 11$), 2(\mathrm{IQR}=0$ to 4$), 9$ (IQR= 2 to 15$)$ and 7 (IQR= 2 to 13 ), respectively. The median of $\mathrm{BRS}$ was 19 ( $\mathrm{IQR}=17$ to 23$)$. Among the participants moderate to severe somatic distress rate was \% 38 and probable PTSD rate was 20.4\%. Depression, anxiety and stress symptoms were $36.1 \%$, $49.1 \%$ and $45.4 \%$, respectively, from mild to extremely severe at any level. The psychometric properties of selfrating questionnaires and cancer patients' severity levels in accordance to questionnaires cutoff points were presented in Table 4.

\section{Comparisons of the somatic distress groups in terms of self-rating inventories}

Kruskal-Wallis test were evaluated to compare differences among somatic symptom severity groups [Minimal (1), Mild (2), Moderate (3), and Severe (4)] in terms of PCL-5 total score , intrusions, avoidance, NACM, hyperarousal, DASS-21-total score, depression, anxiety, stress, BRS, and stressful life events (Table 4).

There were statistically significant differences among somatic symptom severity groups in PCL-5 total score $[\mathrm{H}(3)=32.77, \mathrm{p}<0.001]$, intrusions $[\mathrm{H}(3)=24.60, \mathrm{p}<0.001]$, avoidance $[\mathrm{H}(3)=34.47, \mathrm{p}<0.001], \mathrm{NACM}[\mathrm{H}(3)$ $=22.38, \mathrm{p}<0.001]$, and hyperarousal $[\mathrm{H}(3)=19.58, \mathrm{p}<0.001]$. There were statistically significant differences among somatic symptom severity groups in DASS-21-total score $[\mathrm{H}(3)=56.40, \mathrm{p}<0.001]$, depression $[\mathrm{H}(3)$ $=50.47, \mathrm{p}<0.001]$, anxiety $[\mathrm{H}(3)=60.73, \mathrm{p}<0.001]$, and stress $[\mathrm{H}(3)=34.36, \mathrm{p}<0.001]$. There were statistically significant differences among somatic symptom severity groups in $\mathrm{BRS}[\mathrm{H}(3)=21.20, \mathrm{p}<0.001]$, and stressful life events $[\mathrm{H}(3)=14.46, \mathrm{p}=0.002]$.

\section{Multiple linear regression analyses for somatic distress}

Multiple linear regression was carried out for predicting somatic distress (Table 5). Low educations levels, high anxiety levels, high experience stressful life events, and low psychological resilience predicted high somatic distress levels $(\mathrm{N}=216, \mathrm{R} 2=0.375, \mathrm{~F}(4,211)=31.65, \mathrm{p}<0.001)$. 


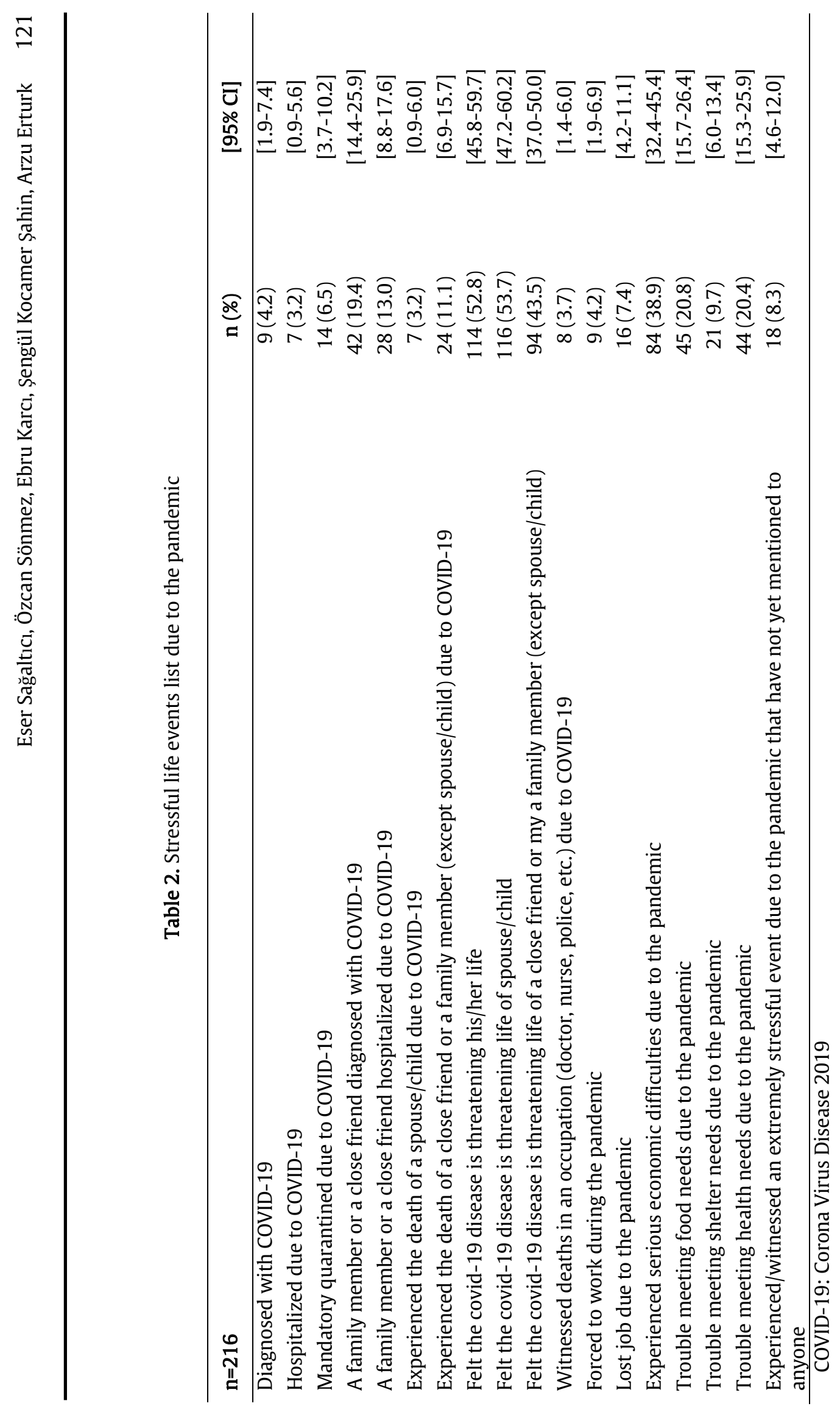




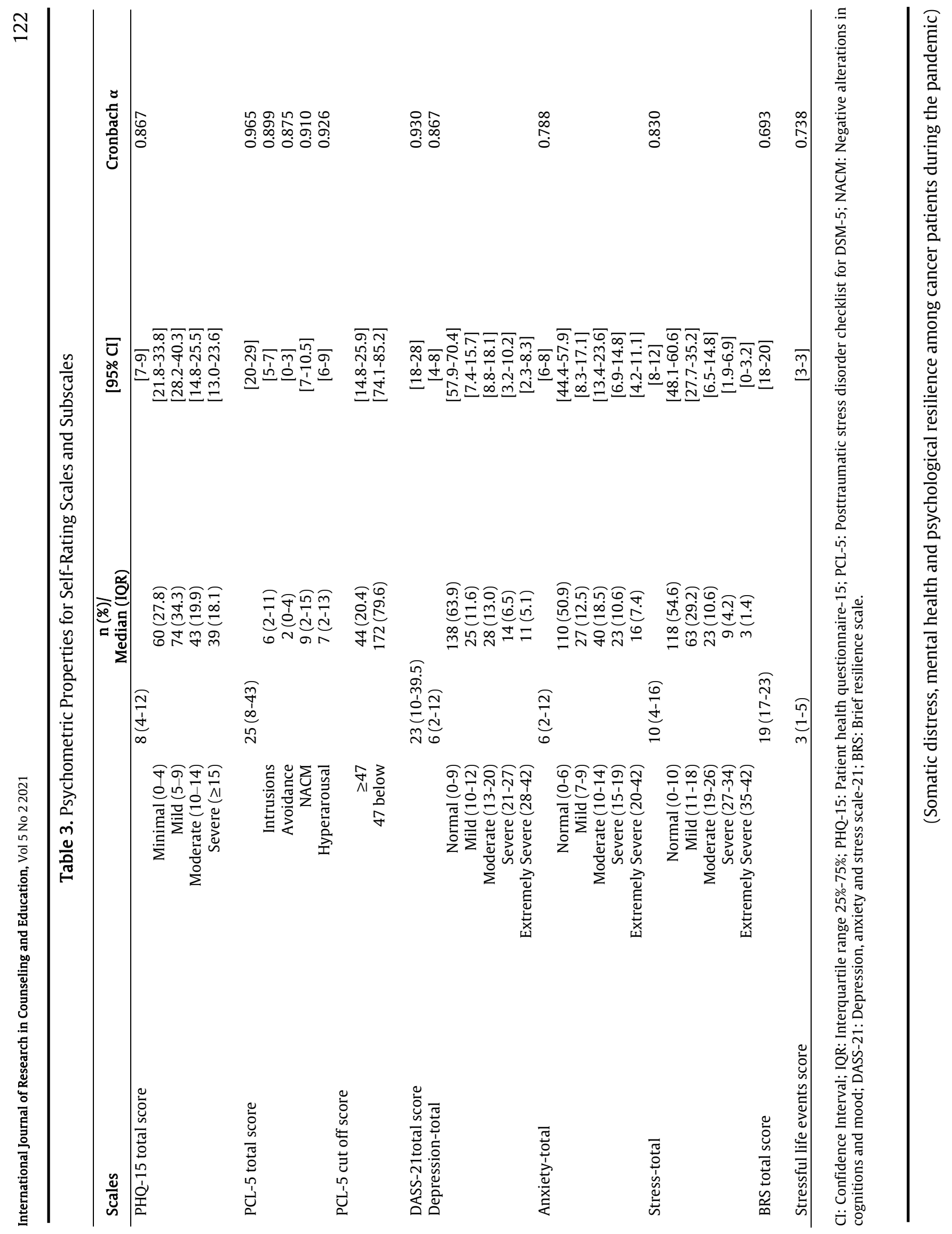




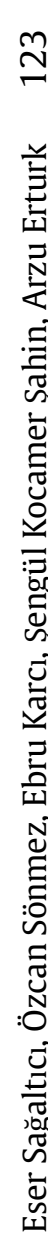
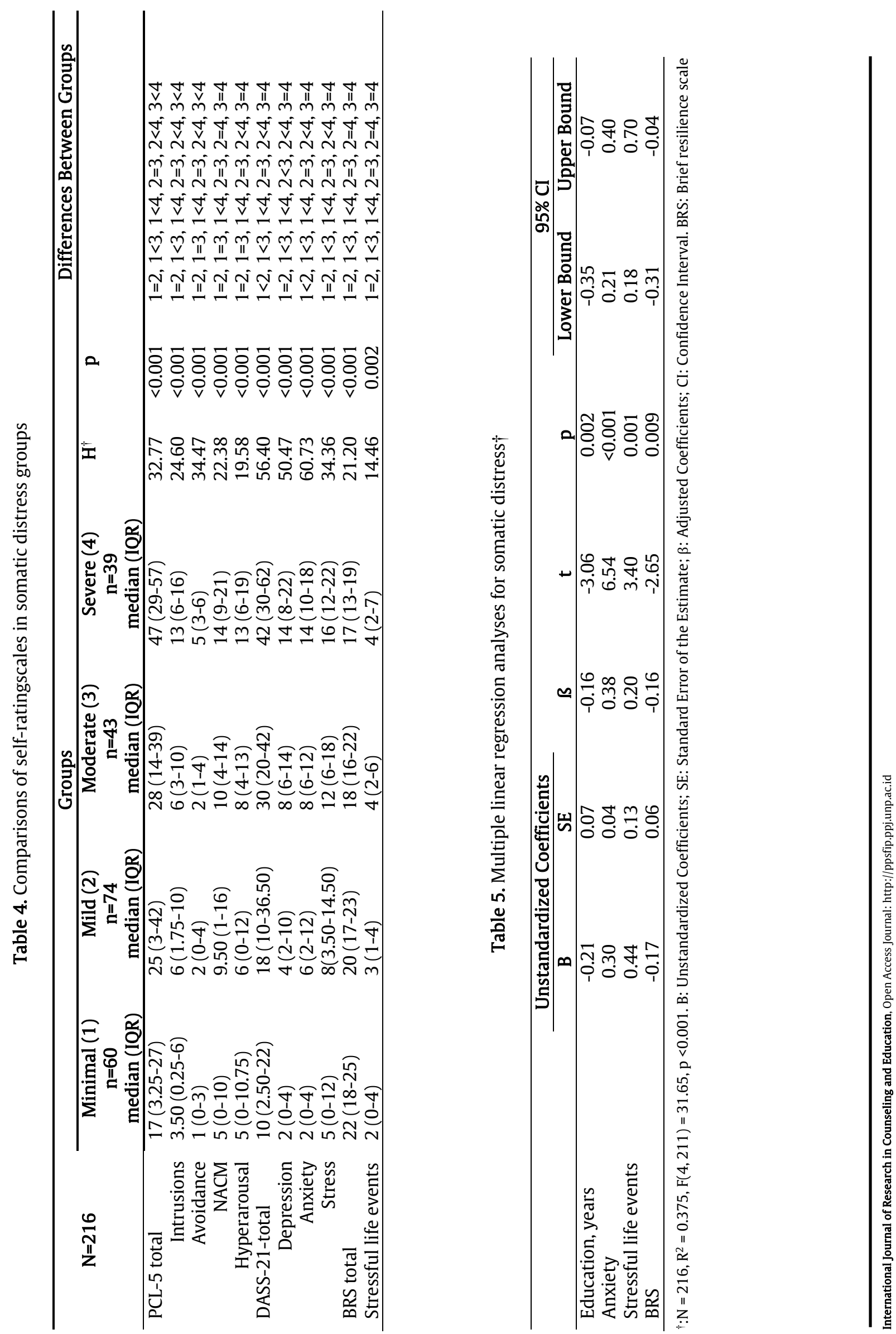
The current researches during COVID-19 have not yet addressed somatic distress among cancer patients. The aims of this study are to investigate the somatic distress, PTSD, depression, anxiety, and stress levels of cancer patients whom already vulnerable population, and analyze the influencing factors on somatic distress during the pandemic. Main findings of this study; among the cancer patients moderate to severe somatic distress rate was \% 38 and probable PTSD rate was 20.4\%. Depression, anxiety and stress symptoms were $36.1 \%$, $49.1 \%$ and $45.4 \%$, respectively, from mild to extremely severe at any level. There were substantial association between somatic symptoms severity and high PTSD, anxiety, depression, and stress symptoms levels. Low educations levels, high anxiety levels, high experience stressful life events, and low psychological resilience predicted high somatic distress levels.

While the evidence of the psychological effects of the COVID-19 pandemic on the general population continues to increase, studies investigating the effects on special and sensitive populations such as cancer patients are gaining momentum. One of the first studies from China, Wang et al. (2020) showed that among 6213 cancer patients, $17.7 \%$ had anxiety, $23.4 \%$ had depression and 9.3\% PTSD symptom. Chen et al. (online) reported that fear of disease progression, anxiety (16.5\%) and depression (28.8\%) was much higher in cancer patients under the COVID-19 outbreak than in the general population and then the cancer patients in times without an outbreak. Juanjuan et al. (2020) found that patients with breast cancer had high rates of anxiety, depression, distress, and insomnia in the peak time of the COVID-19 pandemic. Unlike Musche et al. (2020) found that 150 cancer patients under treatment had similar levels of distress and anxiety to healthy controls during the first days of the pandemic, but cancer patients had more adherent safety behavior. Similar to the majority of those studies, it is seen in our study that patients had high level of anxiety and stress insomuch that they were more afraid of catching COVID-19 (39\%) or not being able to access treatment on time (38\%) because of pandemic than the cancer recurrence (31.5\%) in our study. Schellekens et al. (2020) also showed that patients were most afraid of being infected with COVID-19 (50.5\%) and requiring treatment in the intensive care unit (58.0\%).

To our knowledge, the current study is the first to address COVID-19-related PTSD, depression, anxiety, stress, as well as somatic symptoms (using PHQ) in cancer patients. We found only one study that has closely examined somatic symptoms' association with depression, anxiety and PTSD, focusing solely pain and fatigueas somatic symptoms. However, anxiety and stress, which cause aggravation of somatic symptoms such as pain or fatigue, is a well-studied area Grassi, Caruso \& Nanni 2013; Brown, Kroenke; 2009). Somatic symptoms have already been reported in past pandemics even in general population and psychosomatic responses to these psychological effects should be considered (Chew et al., 2020). Based on this phenomenon, Chaturvedi reported his prediction of the prevalence of chronic pain and the severity of the pain experience may have increased during the COVID-19 pandemic process (Chaturvedi, 2020). Wang et al. (2020) evaluated only physical fatigue and pain intensity as somatic distress in cancer patients using the Visual Analogue Scale (VAS) in their study. They found that pain intensity was a risk factor for anxiety, depression and PTSD. We also found a significant relationship between the severity of somatic symptoms and the levels of anxiety, depression, PTSD and stress symptoms. The fact that patients with high anxiety levels, high stressful life events experience, and low psychological resilience seem most vulnerable to somatic distress among cancer patients' is another significant result of this study.

Anxiety, depression and PTSD is associated with somatic symptoms (Haug, Mykletun \& Dahl, 2004; Bartel et al., 2020). It is thought that the somatic sensory processing mechanism extends to cognitive symptoms and reveals a potential generalization of the impairment in the cognitive and somatic domains in PTSD[31].In line with this, it is seen that 'negative alterations in cognitions and mood' are high in severe somatic distress in PTSD symptomology like other symptomatologies in this study. The COVID-19 outbreak, like other pandemics, was associated with a wide range of psychiatric comorbidities, including anxiety, panic, depression and traumarelated disorders. As a result, cancer patients may be experiencing more somatic distress after all these stressful life events, uncertainty and anxiety during this COVID-19 pandemic.

Psychological Resilience is the ability of an individual to adapt to and cope with adverse situations such as a trauma, threat, health issues (Connor \& Davidson, 2003; Wingo et al., 2010). Resilience is protective against some mental disorders such as anxiety and PTSD while low psychological resilience is risk factor for mental disorders (Pietrzak et al.; 2009). Ran et al. (2020) showed that psychological distress was high at the peak level of COVID-19 outbreak in China, and was negatively correlated with resilience in the general population. Kimhi et al. (2020) showed that individual resilience and well-being were the first and most important predictors of COVID-19 related anxiety. Kavcic et al. (2020) suggested that resilience is of great importance factor that promotes good psychological functioning during COVID-19 outbreak. When these studies are considered together, it is an expected result that high anxiety and low psychological resilience also predict somatic distress in cancer patients during the COVID 19 process.

(Somatic distress, mental health and psychological resilience among cancer patients during the pandemic) 
There are studies that report that less education is associated with greater somatic symptoms (Sayar Kirmayer \& Taillefer, 2003; Zeleke, Minaye \& Kygana; 2015). However, Sayar et al. (2003) reported that low education level predicted somatic symptoms due to comorbidity with depressive symptomology. Zeleke et al. (2015), on the other hand, showed that low education was associated with somatic symptoms in traumatic individuals. So Somatic symptoms may be more apparent in people with low education due to the overlap of COVID-9 stress in cancer patients who are already under own disease stress.

There are several limitations in this study. First, because its cross-sectional design, a cause-effect relationship in variables cannot be established. Whereas, knowing the pre pandemic situation could enable us to reveal the results more clearly. It is a limitation that self-assessment scales were used and additional psychopathologies were not able to screen with a structured interview like SCID. To better understand the impact of COVID-19 on cancer patients as well as somatic distress, large scale studies within the framework of longitudinal and prospective designs are needed.

\section{Conclusions}

This study focused on the psychological outcomes of the COVID 19 outbreak in cancer patients and its relation to somatic distress, which is already a gap in the literature. The results showed that the symptoms of depression, anxiety, stress, and PTSD increased, as well as somatic distress. Moreover, indicators predicting somatic distress were determined as; "'low education levels, high anxiety levels, experience of high stressful life events and low psychological resilience", for which preventive measures should be planned in future studies.

\section{Conflict of interest}

The authors declare that there are no conflicts of interest.

\section{Ethical approval}

All procedures performed in studies involving human participants were in accordance with the ethical standards of the institutional and/or national research committee and with the 1964 Helsinki declaration and its later amendments or comparable ethical standards. The data that support the findings of this study are openly available in [XXX XXXXXX ethics committee], reference number [2020.06.1.11.082].

\section{Informed consent}

Informed consent was obtained from all individual participants included in the study.

\section{Funding source}

This research received no specific grant from any funding agency in the public, commercial, or not-for-profit sectors.

\section{References}

Akin, A. \& Cetin, B.. (2007). The Depression Anxiety and Stress Scale (DASS): The Study of Validity and Reliability. Educ Sci Theory Pract. 7:260-268.

Anuk, D., Özkan, M., Kizir, A. \& Özkan, S. (2019) The characteristics and risk factors for common psychiatric disorders in patients with cancer seeking help for mental health. BMC Psychiatry. 19(1):269.

Bartel, A., Jordan, J., Correll, D., Devane, A \& Samuelson, KW. (2020) Somatic burden and perceived cognitive problems in trauma-exposed adults with posttraumatic stress symptoms or pain. J Clin Psychol. 76(1):146-160.

Boysan, M., Ozdemir., PG, Ozdemir., O., et al. (2017). Psychometric properties of the Turkish version of the PTSD Checklist for Diagnostic and Statistical Manual of Mental Disorders, Fifth edition (PCL-5). Psychiatry Clin psychopharmacology. 27(3):306-16.

Breitbart, W., Rosenfeld, B., Pessin, H., et al. (2000) . Depression, hopelessness, and desire for hastened death in terminally ill patients with cancer. JAMA. 284(22):2907-11.

Brown, LF., \& Kroenke, K. (2009). Cancer-related fatigue and its associations with depression and anxiety: a systematic review. Psychosomatics. 50(5):440-447. 
Chan, JF., Yuan S., Kok, KH., et al. (2020) A familial cluster of pneumonia associated with the 2019 novel coronavirus indicating person-to-person transmission: a study of a family cluster. Lancet. 395(10223):514-523.

Chaturvedi, SK. (2020). Health anxiety, health-related life events, and somatization during COVID-19 pandemic can increase chronic pain. Pain. 161(11):2652.

Chen, G., Wu., Q Jiang, H., et al. (2020). Fear of disease progression and psychological stress in cancer patients under the outbreak of COVID-19. Psychooncology. 2020;10.1002/pon.5451. https://doi.org/10.1002/pon.5451.

Chew, QH., We i., KC, Vasoo., S, Chua, HC., Sim, K. (2020). Narrative synthesis of psychological and coping responses towards emerging infectious disease outbreaks in the general population: practical considerations for the COVID-19 pandemic. Singapore Med J. 61(7):350-356.

Connor, KM. \& Davidson, JR. (2003).Development of a new resilience scale: the Connor-Davidson Resilience Scale (CD-RISC). Depress Anxiety. 18(2):76-82.

Dogan, T. (2015). Kısa psikolojik sağlamlık ölçeği'nin türkçe uyarlaması: Geçerlik ve güvenirlik çalışması [Adaptation of the Brief Resilience Scale into Turkish: A validity and reliability study]. The Journal of Happiness \& Well-Being, 3(1), 93-102.

Eser, HY. (2020). COVID-19 Döneminde Stres, Adaptasyon ve Psikiyatrik Etkiler. Türkiye Klin COVID - 19. $1(13): 18-22$.

González-Montero, J., Valenzuela, G., Ahumada, M., Barajas, O., Villanueva, L. (2020) Management of cancer patients during COVID-19 pandemic at developing countries. World J Clin Cases. 8(16):3390-3404.

Grassi, L., Caruso, R., Nanni, MG. (2013). Somatization and somatic symptom presentation in cancer: a neglected area. Int Rev Psychiatry. 2013;25(1):41-51.

Haug, TT., Mykletun, A., Dahl, AA. (2004). The association between anxiety, depression, and somatic symptoms in a large population: the HUNT-II study. Psychosom Med. 66(6):845-851.

Juanjuan, L., Santa-Maria, CA., Hongfang, F., et al. (2020). Patient-reported Outcomes of Patients With Breast Cancer During the COVID-19 Outbreak in the Epicenter of China: A Cross-sectional Survey Study. Clin Breast Cancer. 20(5):e651-e662.

Kavčič, T., Avsec, A., Zager, KG. (2020). Psychological Functioning of Slovene Adults during the COVID-19 Pandemic: Does Resilience Matter?. Psychiatr Q. https://doi.org/10.1007/s11126-020-09789-4

Kimhi, S., Marciano, H., Eshel, Y., Adini, B. (2020). Resilience and demographic characteristics predicting distress during the COVID-19 crisis. Soc Sci Med. 265:113389.

Kroenke, K., Spitzer, R.L., Williams J.B.W. (2002). The PHQ-15: validity of a new measure for evaluating the severity of somatic symptoms. Psychosom Med. 64:258-266.

Lovibond, P.F. \& Lovibond, S.H. (1995). The structure of negative emotional states: Comparison of the Depression Anxiety Stress Scales (DASS) with the Beck Depression and Anxiety Inventories. Behav Res Ther.33:335-343

Miaskowski, C., Paul, S.M., Snowberg, K., et al. (2020). Stress and Symptom Burden in Oncology Patients During the COVID-19 Pandemic. J Pain Symptom Manage. 60(5):e25-e34.

Musche, V., Bäuerle, A,. Steinbach, J., et al. (2020). COVID-19-Related Fear and Health-Related Safety Behavior in Oncological Patients. Front Psychol. 2020;11:1984.

Pietrzak, R.H., Johnson, D.C., Goldstein, M.B., Malley J.C., Southwick S.M. (2009). Psychological resilience and postdeployment social support protect against traumatic stress and depressive symptoms in soldiers returning from Operations Enduring Freedom and Iraqi Freedom. Depress Anxiety. 26(8):745-751.

Ran, L., Wang, W., Ai, M., Kong, Y., Chen, J., Kuang, L. (2020). Psychological resilience, depression, anxiety, and somatization symptoms in response to COVID-19: A study of the general population in China at the peak of its epidemic. Soc Sci Med. 262:113261.

Romito, F., Dellino, M., Loseto, G. et al. (2020). Psychological Distress in Outpatients With Lymphoma During the COVID-19 Pandemic. Front Oncol. 10:1270.

Saya,r K., Kirmayer. L.J., Taillefer, S.S. (2003). Predictors of somatic symptoms in depressive disorder. Gen Hosp Psychiatry. 25(2):108-114. 
Schellekens, M.P.J, van der Lee, M.L. (2020). Loneliness and belonging: Exploring experiences with the COVID19 pandemic in psycho-oncology. Psychooncology. 10.1002/pon.5459. https://doi.org/10.1002/pon.5459

Smith, B.W., Dalen, J., Wiggins, K., et al. (2008). The brief resilience scale: assessing the ability to bounce back. Int J Behav Med. 15(3):194-200.

Wang, C., Horby ,P.W., Hayden, F.G., Gao, G.F. (2020). A novel coronavirus outbreak of global health concern. Lancet. 395(10223):470-473.

Wang, Y., Duan, Z,. Ma, Z., et al. (2020). Epidemiology of mental health problems among patients with cancer during COVID-19 pandemic. Transl Psychiatry. 10(1):263.

Weathers, F.W., Litz, B.T., Keane, T.M., et al. (2013). The PTSD Checklist for DSM-5 (PCL-5). Scale available from the National Center for PTSD website: http://www.ptsd.va.go.

Wingo, A.P., Fani, N., Bradley, B., Ressler, K.J. (2010). Psychological resilience and neurocognitive performance in a traumatized community sample. Depress Anxiety. 27(8):768-774.

Wolfe, J., Kimerling, R., Brown, P.J., Chrestman, K.R., Levin, K.(1996). Psychometric review of the life stressor checklist-revised. In: Stamm BH, editor. Measurement of Stress, Trauma, and Adaptation. Lutherville, $M D$ : Sidran Press; pp. 198-201.

Wu, Z. \& McGoogan, J.M. (2020). Characteristics of and Important Lessons From the Coronavirus Disease 2019 (COVID-19) Outbreak in China: Summary of a Report of 72314 Cases From the Chinese Center for Disease Control and Prevention. JAMA. 323(13):1239-1242.

Yang, J., Zheng, Y,. Gou, X., et al. (2020) .Prevalence of comorbidities and its effects in patients infected with SARS-CoV-2: a systematic review and meta-analysis. Int JInfect Dis. 94: 91-95.

Yas, S.C., Bildik, F., Aslaner, M.A., et al. (2020). The Effect of the COVID-19 Pandemic on the Psychological Status of Hospital Workers. Psychiatry Clin Psychopharmacol.30:264-272.

Yazici, G.M., Güleç, H., Simșek, G., Turhan, M., Aydin, S.E. (2012). Psychometric properties of the Turkish version of the Patient Health Questionnaire-Somatic, Anxiety, and Depressive Symptoms. Compr Psychiatry. 2012;53(5):623-9.

Zelek ,W., Minaye, A., Kygana, G. (2015). Mental Health and Somatic Distress among Ethiopian Migrant Returnees from the Middle East. Int J Ment Health Psychiatry. 1:2. 